\title{
Modeling and Design of a Peak Power Tracking System for Solar Array Generators of Artificial Satellites
}

\author{
E.E. Renato Oliveira de Magalhaes* \\ National Institute for Space Research, Sao Jose dos Campos, SP, 12245-970, Brazil \\ Dr. Marcelo Lopes de Oliveira e Souza ${ }^{\dagger}$ \\ National Institute for Space Research, Sao Jose dos Campos, SP, 12245-970, Brazil
}

\begin{abstract}
The demand for optimal performance is at the core of space system design. In this work a system comprised of a solar array generator supplying power to a Li-Ion battery and several payloads of a space vehicle is analyzed. The goal of this paper is to define the topology of such system, its modes of operation and to model the Peak Power Tracking mode by means of digital control techniques. The validation is done by a hardware prototype which was built and tested to obtain the system dynamic response.
\end{abstract}

\section{Nomenclature}

$\begin{array}{ll}S A G & \text { Solar Array Generator } \\ P C U & \text { Power Conditioning Unit } \\ P D U & \text { Power Distribution Unit } \\ B A T & \text { Battery } \\ P P T & \text { Peak Power Tracking } \\ P_{o} & \text { Optimum Power of Solar Array Generator } \\ P_{s a} & \text { Solar Array Generator Instantaneous Power } \\ V_{e o c} & \text { Battery End of Charge Voltage } \\ I O D S & \text { Indicated Output Deviated Signal } \\ S Z & \text { Stabilization Zone } \\ D C / D C & \text { DC/DC converter } \\ C S & \text { Correction Signal } \\ d i r & \text { Direction of Control Voltage Variation } \\ i_{s a} & \text { Solar Array Generator Current } \\ v_{s a} & \text { Solar Array Generator Voltage } \\ v_{b u s} & \text { Bus Voltage } \\ i_{b u s} & \text { Bus Current } \\ i_{b a t} & \text { Battery Current } \\ v_{c} & \text { Control Voltage } \\ d & \text { Duty Cycle }\end{array}$

\section{Introduction}

GATELlite power supply systems very often makes use of sunlight as a source for electrical energy conSversion. The search for optimal use of photovoltaic cells has lead to many different types of PPT algorithms. ${ }^{3-5,7}$ At the same time the control of physical systems with a digital computer is becoming more and more common. Among the advantages of digital logic is the flexibility of the control programs and

*Electronics Engineer, Aerospace Electronics Division, DEA, non Member.

${ }^{\dagger}$ Researcher, Division of Space Mechanics and Control, DMC, Senior Member. 
the decision-making or logic capability which can be shared with the control function to meet other system requirements. ${ }^{1}$ This is specially true in satellite systems where there is a tendency to incorporate more onboard computing and raw data processing. ${ }^{9}$ The use of microcontroller to perform all these functions also makes easy the implementation of PPT algorithms. In this work we will be modelling such algorithm and obtaining its experimental dynamic response. The simplicity of software will be demonstrated by showing that it does not need too many lines of code. In reality, a PPT system must function in different modes of operation other than the PPT mode. These modes, although considered in the final prototype, are not presented in this work. We will be concerned with the modeling of PPT one only.

Satellite power supply systems comprise the following main functions as stated bellow and shown in figure 1:

- Electrical energy source(SAG): usually a photovoltaic solar panel.

- Electrical energy storage unit(BAT): usually a battery.

- Power conditioning unit(PCU): usually DC/DC converters with control electronics.

- Power distribution and protection unit(PDU): interfaces the payload and the satellite power bus.

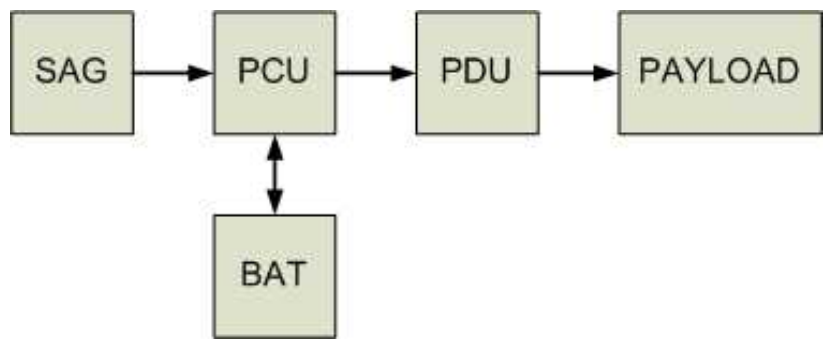

Figure 1. General Architecture of a Power Supply System.

\section{Basic Concepts}

Basically there are two types of power supply system topologies. They can be divided in: direct energy transfer or peak power tracking. ${ }^{9}$ The former connects the solar array generator directly to the satellite bus; the latter drives the output power of the SAG towards its optimum point which corresponds to the maximum power available from the solar array. The topology selected for this work is shown in figure 2

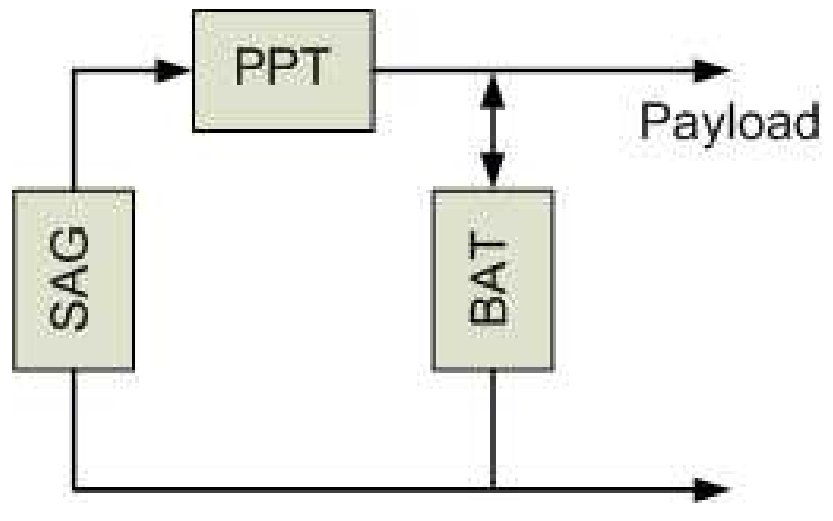

Figure 2. Peak Power Tracking Topology.

In this topology the power supply system delivers a non regulated bus to the payload. The system tracks the maximum power point of the solar array whenever the battery is not fully charged. Once the battery has reached its final state of charge the system must switch from the PPT mode of operation to a mode that regulates the end of charge voltage of the battery without cause any damage to it. During the battery end 
of charge mode the plant is controlled by the appropiate controller compensation. During PPT mode there is no need for a plant transfer function. There is only the need for a characterstic curve that exhibits an optimum point. The type of algorithm presented in this work proved to be robust against variation of system parameters. $^{2}$ This is specially interesting for satellite systems since it faces great variations throughout its orbit cycles. As shown in figure 3 the PPT block is composed of a digital controller that generates a control signal to the input of a modulator whose function is to convert this signal to the desired PWM duty cycle of the $\mathrm{DC} / \mathrm{DC}$ converter. In this fashion one can drive the solar array generator voltage towards the optimum point. The signals $i_{s a}, v_{s a}$ e $v_{b u s}$ are fed back to the multiplexed inputs of an A/D converter by means of current and voltage sensors. The actuation on the plant is achieved by generating a controlling signal $v_{c}$ that modulates the PWM of the DC/DC converter.

The system detailed block diagram is shown in figure 3:

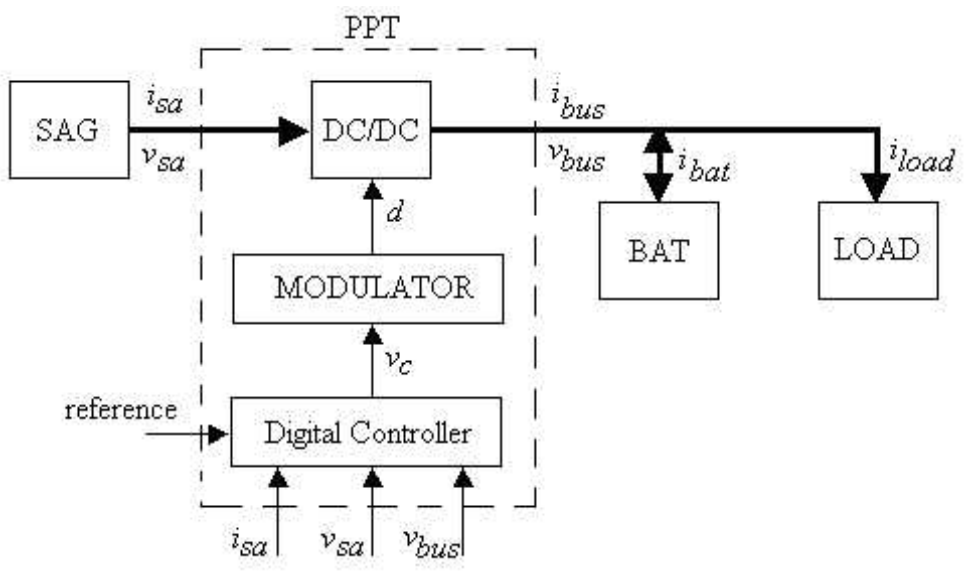

Figure 3. System Detailed Block Diagram.

\section{PPT Mode Modeling}

This paper presents a PPT control technique based on optimal control systems of the peak holding type. In this case it is unecessary to build particular input-output functions into control equipments of this type because it automatically compensates for changes in the controlled system. This control technique also proved to be very robust against system parameter variations and interferences. ${ }^{2}$ In systems of the peak holding type the correction signal $C S$ is based on the difference between an indicated solar array optimum power $P_{o}$ as a reference quantity and the instantaneous solar array power $P_{s a}$ as the compared quantity. The correction signal is generated to vary with the time integral of this error signal. The indicated solar array optimum power $P_{o}$ is carried along with the solar array instantaneous power $P_{s a}$ so long as this signal magnitude is increasing, and remains constant at the highest level reached when the signal $P_{s a}$ starts to decrease. When the magnitude of the correction signal $C S$ reaches the threshold limit established by the stabilization zone $(S Z)$ the controlling signal $v_{c}$ is reversed to drive the $P_{s a}$ signal toward the optimum point $P_{o}$. This reversion takes place whenever the correction signal exceeds the stabilization zone limit. This results in a series of cycles in which the $P_{s a}$ signal is varied within the range delimited by the sabilization zone as the controlling signal changes along a series of straight lines with the rate variation either going up or down. The block diagram that models the algorithm of peak power tracking is presented in figure 4 . An analog to digital converter is used to acquire the voltage and current of the solar array generator. The instantaneous solar array power $P_{s a}$ is obtained by multiplying these two signals. The corresponding curves are shown in figure 5 . The Duty Cycle Generator Block operates to change the solar array voltage $v_{s a}$ at a constant rate, initially positive, as indicated in figure 5. According to the characteristic curve of the solar array this causes the $P_{s a}$ signal to increase and the indicated optimum power $P_{o}$ remains identical with $P_{s a}$. When the magnitude of $P_{s a}$ passes the peak corresponding to the optimum point and starts to decrease, the peak signal is held by the Peak Holder block which works as a kind of optimum power estimator. At this moment the error signal $I O D S$ is generated and its time integral corresponds to the correction signal which in turns feeds the Duty Cycle Generator Block. This block also receives the stabilization zone setting. 


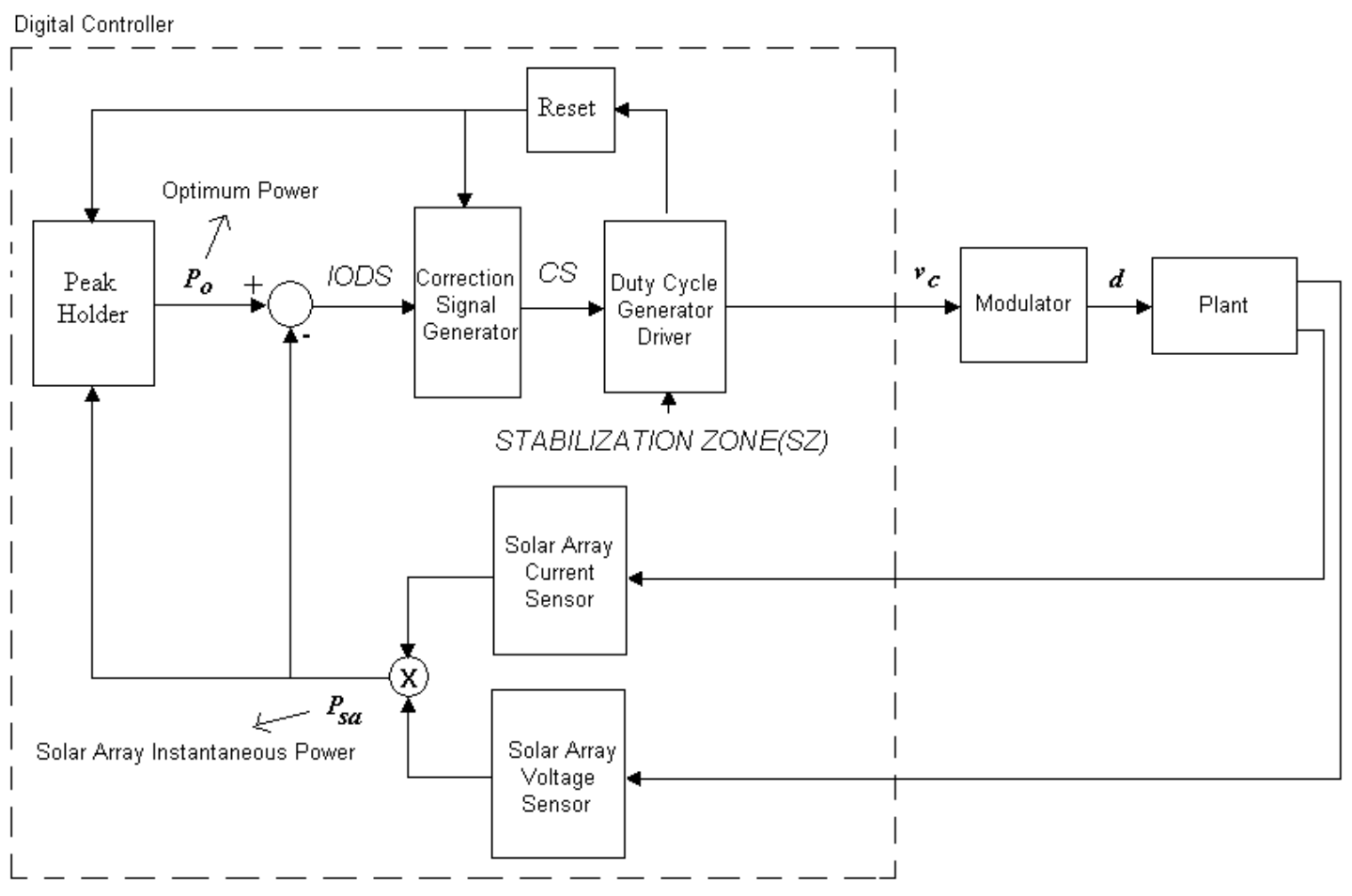

Figure 4. Block Diagram Model of Control Law.

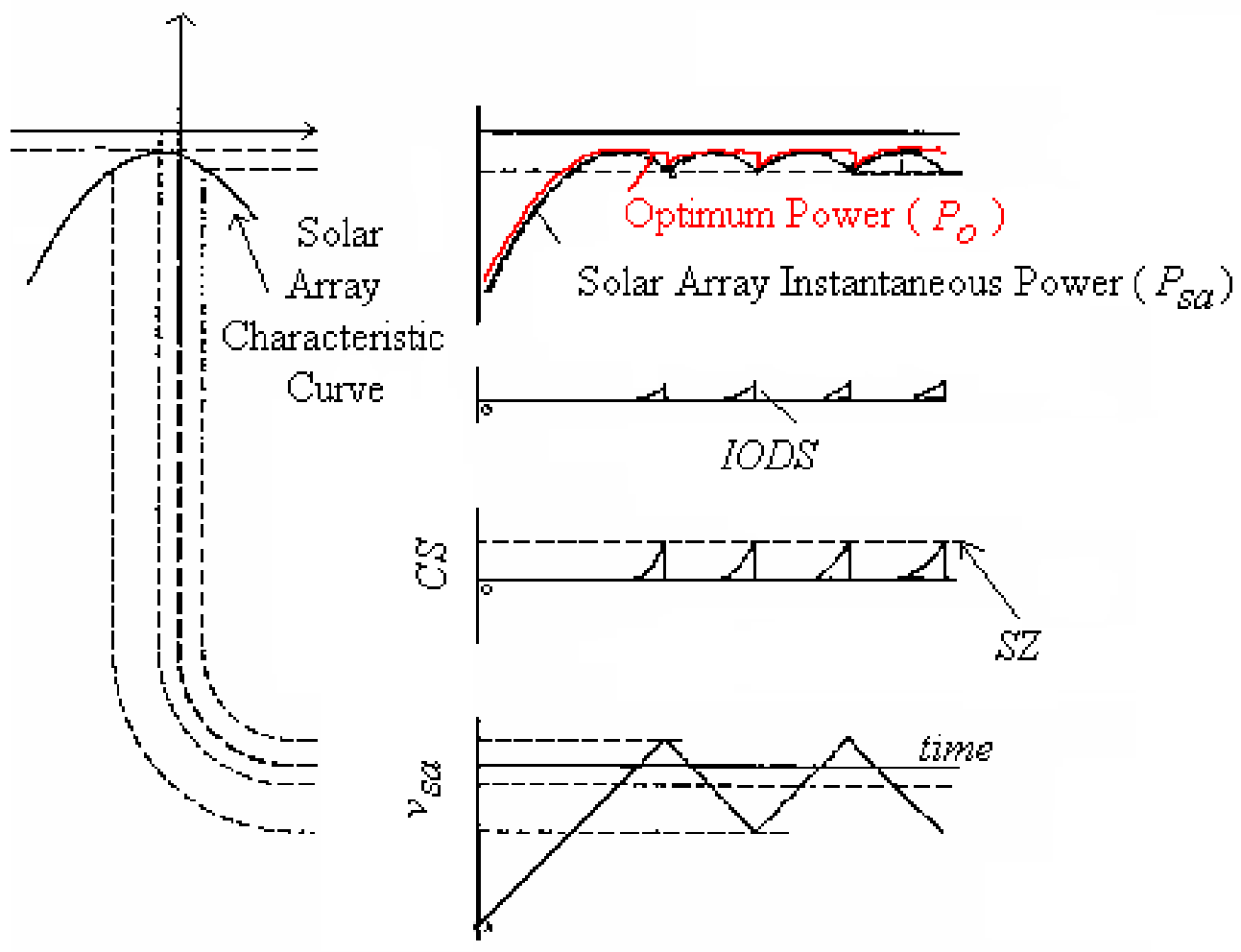

Figure 5. PPT Signals. 
The output of this block is the controlling signal $v_{c}$. At each of the controlling signal reversal times this block generates a reset signal to the Peak Holder and Correction Signal Generator Blocks. This reset signal causes the IODS signal to be reduced to zero and the indicated optimum power $P_{o}$ to be made equal to the instantaneous power $P_{s a}$. Therefore the integral that is used to generate the correction signal also is made zero again at each instant of reversal time.

\section{Experimental Results}

A prototype was built to validate the model previously described. The electrical diagram is presented in the appendix section. It uses a 8031 microcontroller operating at $5 \mathrm{Mhz}$ clock to generate a discrete control law which is converted to an analog signal by means of a D/A converter. The DC/DC converter uses a buck topology with PWM frequency selected at 150Khz. The battery technology used was Li-Ion type and the payload was simplified by a voltage controlled current source implemented by a MOSFET. The software flowchart is also presented in the appendix section and we can see that the microcontroller implements most of the algorithms functions. Therefore the multiplication of solar array current and voltages, peak holding, error and correction signal are all inside the software which simplifies the external circuitry. The result software code was no longer than two hundred lines when written in assembly language. Looking at figure 6 one can see that as we vary linearly the solar array voltage around its optimum point the solar array current swings in a parabolic shape. The frequency of PPT searching mechanism can be adjusted by changing the rate that the controlling voltage $v_{c}$ is varied. The PPT efficiency mechanism is controlled by the parameter stabilization zone $(S Z)$. This efficiency is related to the percent ratio between the instantaneous solar array power at time reversal and its optimum power. This defines the output hunting zone and one wishes to keep it as low as possible while maintaining the system stable.

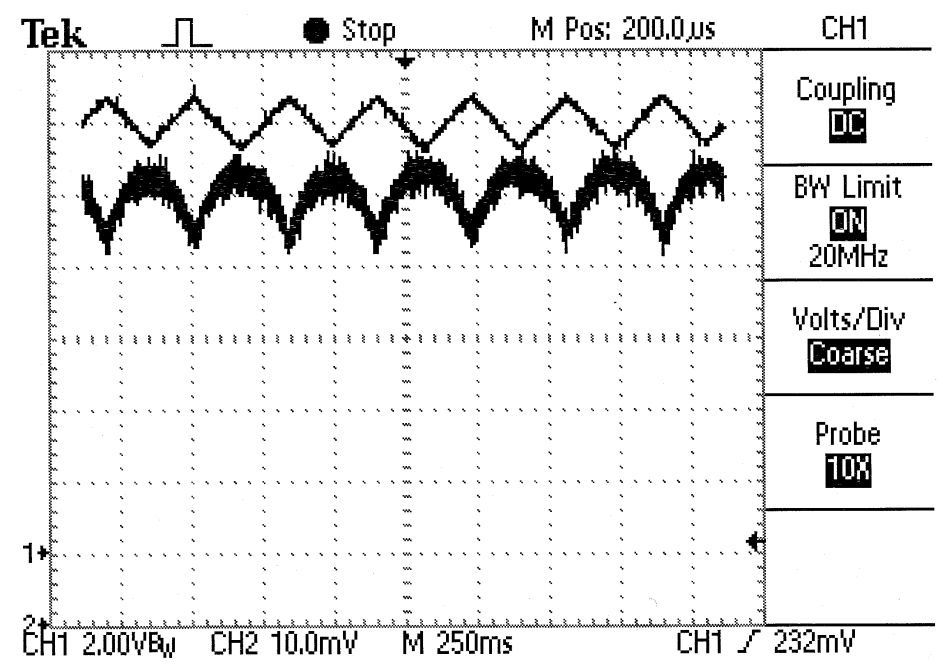

Figure 6. Experimental Results. CH1(top curve,2V/div.) is solar array voltage and CH2(bottom curve, $100 \mathrm{~mA} /$ div.) is solar array current.

\section{Conclusion}

This paper presented a power supply system topology and the modeling of one of its modes of operation. The control of the system is of the digital type and its function is the maximum peak power tracking of a solar array generator. The implementation of a digital control law made use of a 8031 microcontroller and the result software complexity was shown to be little with less than two hundred lines of assembly code. 


\section{Appendix}

\section{A. Software Flowchart}

As stated before the control of this system makes use of digital techniques by means of a 8031 microcontroller. All the controlling functions are done by this component with the aid of $\mathrm{A} / \mathrm{D}$ and $\mathrm{D} / \mathrm{A}$ converters. The flowchart shown in figure 7 basically implements the functions described by the block diagram discussed before.

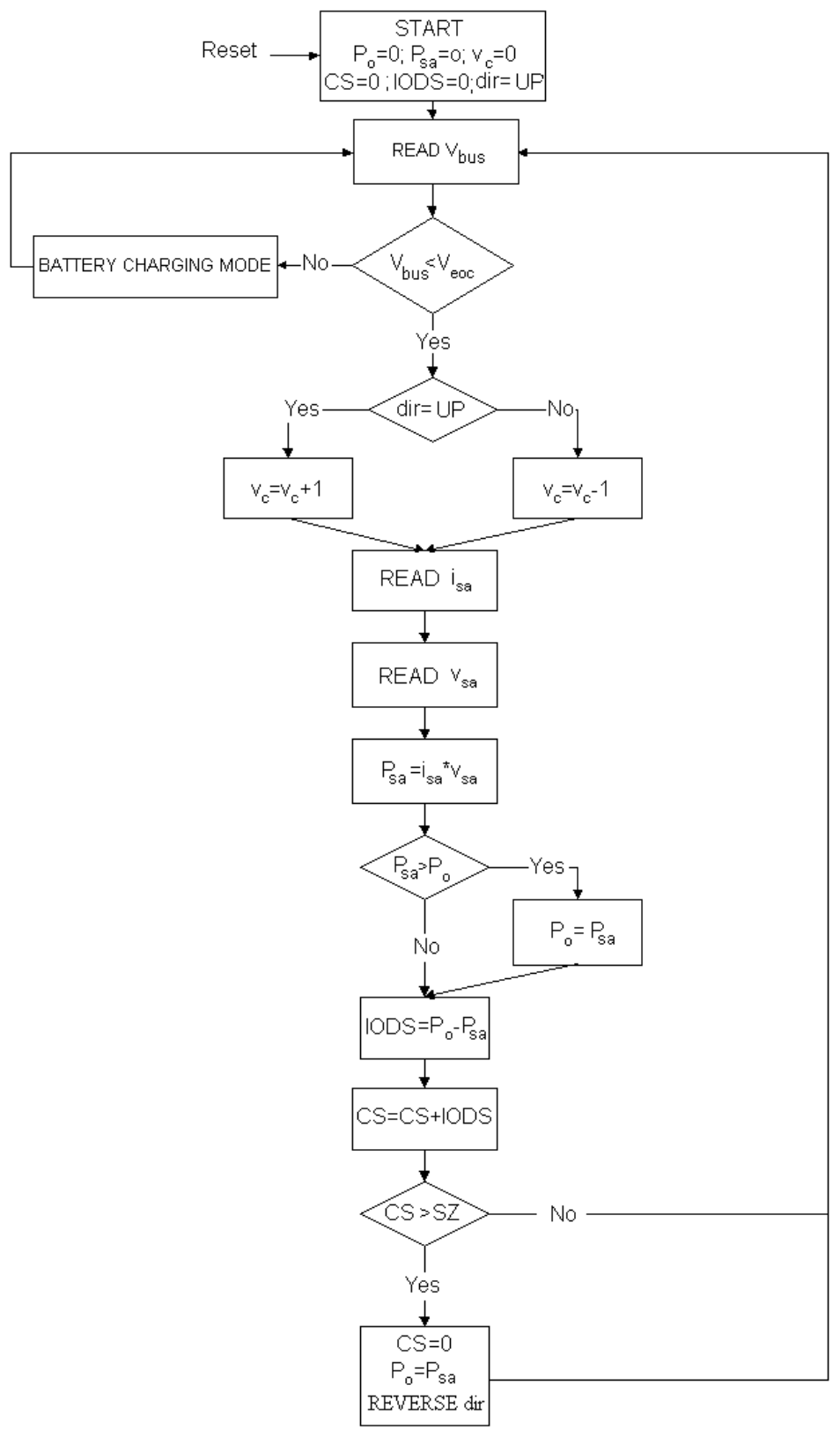

Figure 7. System Detailed Block Diagram.

The variable dir controls the diretion of the controlling voltage rate. That is, if dir is equal to UP then the signal $v_{c}$ has a positive slope. Whenever the system reaches the threshold established by the stabilization zone, it reverses the variable dir and the controlling voltage decreases with time with this process repeating for each cycle of peak power hunting. 


\section{B. Electrical Diagram}

Figure 8 shows the electrical diagram that corresponds to the prototype used to validate the modes of operation of the system. The set comprised by the $\mathrm{A} / \mathrm{D}$, microcontroller and $\mathrm{D} / \mathrm{A}$ is simplified in the diagram to give the idea of its main function only.

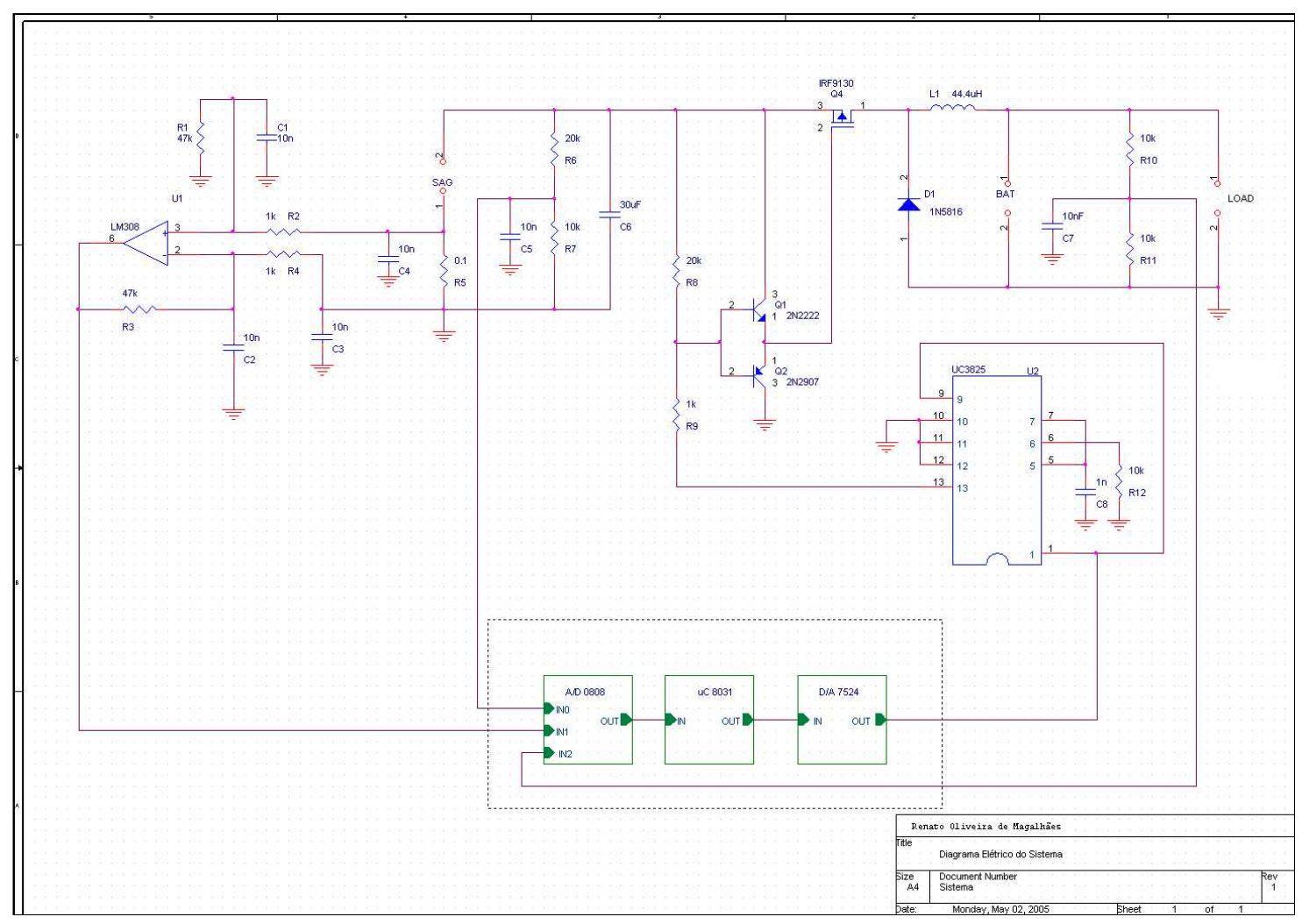

Figure 8. Electrical Diagram.

\section{Acknowledgments}

The authors thanks the National Institute for Space Research for the laboratory infrastructure.

\section{References}

\footnotetext{
${ }^{1}$ Franklin, G. F. and Powell, J. D., Digital Control of Dynamic Systems,Reading, MA, USA, Addison-Wesley Pub. Co.,Inc, 1980.

${ }^{2}$ Draper, C. S. and Li, Y. T., Principles of Optimilizing Control Systems and Application to the Internal Combustion Engine, The American Society of Mechanical Engineer, New York, 1951.

${ }^{3}$ Huynh, P. T. and Cho, B. H., Design and Analysis of a Regulated Peak Power Tracking System, 30th Intersociety Energy Conversion Engineering Conference-IECEC, pp285-290, 1995.

${ }^{4}$ Caldwell, D. J.,Bavaro, L. T. and Carian,R. J., Advanced Space Power System with Optimized Peak Power Tracking, Intersociety Energy Conversion Engineering Conference, vol.2, pp145-150, 1991.

${ }^{5}$ Tevlings, W. J. A., Marpinard, J. C., Capel, A. and Sullivan, D. O., New Maximum Power Point Tracking Systems, 24th Annual Power Electronics Specialist Conference, p833-838, 1993.

${ }^{6}$ Freeman, W. E., Peak Power Tracker Versus Direct Energy Transfer Electrical Power Systems, Intersociety Energy Conversion Enegineering Conference, vol. 2, p387-391, 1992.

${ }^{7}$ Huinh, P. and Cho, B. H., Design and Analysis of a Microprocessor-Controlled Peak-Power-Tracking System, IEEE Transactions on Aerospace and Electronics Systems, vol. 32, no. 1, pp182-190, 1996.

${ }^{8}$ Cho, B. H., Lee, J. R. and Lee, F. C. Y., Large-Signal Stability Analysis of Spacecraft Power Processing Systems, IEEE Transactions on Power Electronics, vol. 5, no. 1, pp110-116, 1990.

${ }^{9}$ Larson, W. J. and Wertz, J. R., Space Mission Analysis and Design, $2^{\text {nd }}$ ed., Microcosm, Inc., Torrence, CA, 1992.
} 